\title{
Oxytocin and mutual communication in mother-infant bonding
}

\author{
Miho Nagasawa, Shota Okabe, Kazutaka Mogi and Takefumi Kikusui *
}

Department of Animal Science and Biotechnology, Azabu University, Sagamihara, Kanagawa-ken, Japan

\section{Edited by:}

Bert Timmermans, University

Hospital Cologne, Germany

Reviewed by:

Rebecca Elliott, University of

Manchester, UK

Jack van Honk, Utrecht University,

Netherlands

*Correspondence:

Takefumi Kikusui, Department of

Animal Science and Biotechnology,

Azabu University, 1-17-71

Fuchinobe, Chuoh-ku, Sagamihara,

Kanagawa-ken 252-5201, Japan.

e-mail:kikusui@azabu-u.ac.jp
Mother-infant bonding is universal to all mammalian species. In this review, we describe the manner in which reciprocal communication between the mother and infant leads to mother-infant bonding in rodents. In rats and mice, mother-infant bond formation is reinforced by various social stimuli, such as tactile stimuli and ultrasonic vocalizations (USVs) from the pups to the mother, and feeding and tactile stimulation from the mother to the pups. Some evidence suggests that mother and infant can develop a cross-modal sensory recognition of their counterpart during this bonding process. Neurochemically, oxytocin in the neural system plays a pivotal role in each side of the mother-infant bonding process, although the mechanisms underlying bond formation in the brains of infants has not yet been clarified. Impairment of mother-infant bonding, that is, deprivation of social stimuli from the mother, strongly influences offspring sociality, including maternal behavior toward their own offspring in their adulthood, implying a "non-genomic transmission of maternal environment," even in rodents. The comparative understanding of cognitive functions between mother and infants, and the biological mechanisms involved in mother-infant bonding may help us understand psychiatric disorders associated with mother-infant relationships.

Keywords: mother-infant, bonding, oxytocin, maternal behavior

\section{INTRODUCTION}

"Sympathy is much strengthened by habit. In however complex a manner this feeling may have originated, as it is one of high importance to all those animals which aid and defend one another, it will have been increased through natural selection; for those communities, which included the greatest number of the most sympathetic members, would flourish best, and rear the greatest number of offspring" (Charles Darwin, "Descent of Man," 1871).

During the process of mammalian evolution, animals developed sympathetic neural and behavioral systems, in which for example, weak and helpless member of individuals are protected and nurtured by other group members. This phenomenon is mostly clearly observed in mother-infant relationship, such as mother infant bonding (Broad et al., 2006).

Social bonds like mother-infant bonding are hypothetical constructs and cannot be measured directly. However, there are several behavioral and physiological measures that have been used as indices of social bonding, including increased physical proximity (Hennessy, 1997), behavioral distress, or elevated corticosteroid levels following separation from the bonding partner (Ziegler et al., 1995; Norcross and Newman, 1999). Social bonding has not yet been clearly defined, but it has been proposed that social bonding can be distinguished neurochemically from social affiliation, in which corticosteroid elevation does not occur following separation (DeVries, 2002). Moreover, subsequent reunion with conspecific animals ameliorates separation distress or aversive experiences. This phenomenon is termed as "social buffering"
(Kikusui et al., 2006); its effect depends on the degree of affiliation with the partner and is strongest with the bonding partner, such as that seen in the dyad of mother-infant.

Mother-infant bonding is unique with respect to its influence on the offspring's future. This idea was first suggested in humans by Bowlby's attachment theory (Bowlby, 1969). Subsequently, many psychological and animal research studies have reported that child abuse or childhood neglect are correlated with severe, deleterious long-term effects on the child's cognitive, socioemotional, and behavioral development (Hildyard and Wolfe, 2002). The developmental effects of mother-infant bonding have also been indicated experimentally in non-human primates. For example, in a study by Winslow et al. (2003), mother-reared and human nursery-reared monkeys were subjected to a novel environment with or without a cage mate. The monkeys reared by their mothers exhibited a reduced cortisol response when a social partner was available, whereas nursery-reared monkeys did not. In nursery-reared monkeys, social contact, such as allogrooming and inter-male mounting, was drastically reduced. These findings suggest that the social buffering effect is impaired as nursery-reared monkeys had experienced less social contact in a novel environment. Thus, impairment of mother-infant bonding strongly influences offspring sociality in human and non-human primates (Agid et al., 1999; Heim and Nemeroff, 2001), although details of the underlying mechanisms are not yet fully understood. Additionally, because the bonding formation is established during the process of social communication between mother and infants, social cognition has a pivotal influence on the bonding process 
(Ross and Young, 2009). However, little information has been obtained regarding the role of each social cue in the formation of bonds.

In the present review, we describe the manner in which mutual communication between mother and infant leads to motherinfant bonding in rodents. We emphasize the significance of the conserved oxytocin neural system in mother-infant bond information, with several studies having shown that oxytocin plays a fundamental role in establishing this bond (Kendrick, 2000; Young et al., 2001; Wang and Aragona, 2004; Young and Wang, 2004). Other neurotransmitters that regulated social bonding, such as opioids and dopamine are also important, however, we would concede these issues in other articles. We also review the effects of deprivation of mother-infant bonding, by studying the consequences of early weaning on neurobehavioral development in rodent offspring. Intensive maternal care has evolved and has been preserved, uniquely in mammals, and it is highly probable that mother-infant bonding is universal to all mammalian species. These comparative points of view provide insights into the biological significance of mother-infant bonding in mammals; a comparative understanding of the developmental consequences of this bonding and its underlying mechanisms, even in rodents, may help in our treatment or prevention of disorders associated with child abuse or childhood neglect (Agid et al., 1999; Heim and Nemeroff, 2001).

\section{SOCIAL CUES FROM INFANTS TO MOTHER}

Although rodent pups have limited thermoregulatory and physical capabilities during the first 1 or 2 postnatal weeks, they produce a variety of signals to their mother, such as olfactory and auditory signals (Levy et al., 2004; Ehret, 2005). These social cues play a very important role of facilitating maternal care and ensuring formation of the mother-infant bond.

Ultrasonic vocalizations (USVs) are among the repertoire of auditory outputs from rodent pups. When infant rodents are isolated from their mother and/or littermates, they cool rapidly and emit USVs (Branchi et al., 2001; Ehret, 2005). The incidence of pup USVs generally increases during the first 6-7 days of life. These vocalizations peak around day 8 , after which they start to decrease, finally disappearing at 2 weeks after birth (Elwood and Keeling, 1982). Although vocalizations ranges differ between mice and rats, (the range of mice was approximately $30-80 \mathrm{kHz}$, and that of rats was approximately $30-50 \mathrm{kHz}$ ), many studies have shown that pup USVs could stimulate maternal behavior such as searching for/retrieving pups in both species (Brown, 1973; Branchi et al., 2001). In a previous study, we showed that mother mice responded to pup USVs reproduced by ultrasonic speakers, but they did not respond to other synthesized ultrasounds such as double-duration USVs (Uematsu et al., 2007), indicating that mother mice can recognize the specific pattern of pup USVs in order to retrieve their pups and return them to the nest. Moreover, we showed that social experiences such as mating or parenting enhanced both retrieving behavior and the responses to pup USVs (Okabe et al., 2010). These results indicate that the mother mice showed specific responsiveness to pup USVs, and this sound contributed to the induction of maternal behavior. Interestingly, electrophysiological studies reported that the auditory cortex of mother mice showed specific activation in response to pup USVs, as compared to non-experienced (those having never had a litter) female mice (Liu and Schreiner, 2007; Cohen et al., 2011). Therefore, maternal behavior expression can be at least partially regulated by functional changes in the sensory cortical representation of pup USVs. However, there is a critical missing link between cortical responses to pup USVs and expression of maternal behavior, which is probably regulated by neurons in the medial preoptic area (mPOA) of the hypothalamus (Numan, 2007).

Olfactory cues from pups were also found to be extremely important in stimulating maternal behavior in rodents. There have been many studies showing the behavioral or neural responses to pup odor, and it was shown that the role of olfactory inputs in maternal behavior is different between rats and mice (Levy et al., 2004). In rats, olfactory stimuli clearly inhibit maternal behavior before parturition, a phenomenon termed neophobia. After parturition, however, mother rats drastically change their behavior; they develop a high level of responsiveness to olfactory cues from their pups, and show intensive maternal behavior toward pups (Fleming et al., 1989; Kinsley and Bridges, 1990). This suggests that the experience of partum and the hormonal changes accompanying pregnancy and partum induce this maternal behavior. Once they start displaying maternal behavior, mother rats show a high preference for bedding soiled by pups over clean bedding (Kinsley and Bridges, 1990). In addition, when the pups' heads were rubbed with their anogenital smears and the anogenital areas were cleaned, the dams licked only the head region (Brouette-Lahlou et al., 1991), suggesting that odors emitted from the anogenital area stimulate licking and grooming behavior in the mother rats. Although the behavioral evidence clearly shows that olfaction plays an important organizational role in the induction of maternal behavior, lesions of the main and the accessory olfactory system, or preventing access to the olfactory cues from the pups, produces contradictory or inconsistent results. Several studies have shown that pup retrieval was somewhat impaired by lesions to the olfactory mucosa caused by zinc sulfate (Benuck and Rowe, 1975). However, other studies showed that these manipulations did not decrease maternal behavior (Jirik-Babb et al., 1984; Kolunie and Stern, 1995). Similarly, removal of the vomeronasal organ, such as vomeronasal nerve cuts or olfactory bulbectomy, did not fully disrupt maternal behavior (Jirik-Babb et al., 1984; Morgan et al., 1992). These results suggest that, in rats, olfaction is not crucial for the successful care of pups since the anosmic mother apparently easily compensates for the loss of this sensory input.

Olfactory stimuli appear to play a more significant role in mice than in rats. Mother mice failed to show any maternal behavior following bulbectomy, or anosmia induced by either nasal irrigation of zinc sulfate or by depletion of noradrenaline within the main olfactory bulb (Levy et al., 2004). Moreover, Wang recently showed that type 3 adenylyl cyclase (AC3), which is one among a multigene family of odorant receptors, knockout mothers did not show any pup retrieving or nest building behavior at all (Wang and Storm, 2010). From these lesion and genetic deletion studies, it can be surmised that olfactory stimuli are essential for the induction and maintenance of maternal behaviors in mice. Interestingly, in female mice which had a previous 
maternal experience, pup-killing induced by peripheral anosmia was prevented (Seegal and Denenberg, 1974), indicating that the primiparous mother depends profoundly on olfactory information for the induction of maternal behavior, as compared with the multiparous mother. However, experienced mother mice are able to use multiple sensory inputs and compensate for the loss of one type of sensory cues and still show maternal behavior.

In both mice and rats, maternal behavior is not regulated by a single sensory input. Mothers can use both auditory and olfactory cues to identify and locate their pups. For example, rat mothers can detect pup USVs, and these pup USVs serve as a potent stimulus for the induction of maternal retrieving behavior, particularly when presented together with pup odors (Farrell and Alberts, 2002). Therefore, the integration of multisensory stimulus inputs from pups triggers discrimination and retrieving of pups. Cohen recently showed that exposure to pups' body odor enhances the neuronal response to pup USVs in the primary auditory cortex of mother mice (Cohen et al., 2011). Additionally, they showed that the pup retrieving behavior of mother mice decreased when the pups were washed with warm water (Cohen et al., 2011).

In summary, multisensory stimuli from pups activates the full range of maternal behavior, although there are no current reports of direct anatomical or neural mechanisms that could explain this multisensory integration. Further studies of the neurobiological mechanisms for the integration of olfactory, auditory, and other social stimuli may lead to a better understanding of the role of sensory modalities to induce maternal behavior, which would strengthen the bond between mothers and their pups.

\section{SOCIAL CUES FROM MOTHER TO INFANT}

The neonatal infants are innately attracted to their mother. For instance, odors emitted from the nipple area have a specific character that attracts both rodent and human new-born infants (Porter and Winberg, 1999). Therefore, the infant can suckle the nipple just after the birth after which infant animals can recognize their own mother using chemicals cues, probably because the infant can memorize the mother's individual odors associated with suckling (Moriceau and Sullivan, 2004). This type of odor memory is regulated by the neuropeptide oxytocin (Nowak et al., 2000, 2011). Oxytocin is released in the infant brain while suckling, and it acts on the olfactory bulb. In the olfactory bulb, oxytocin can enhance memory-related neural activation through long-term potentiation (Fang et al., 2008). Therefore, odors from the mother have a fundamental ability to attract her infants.

Physical and tactile stimuli from mother to infants have been indicated to have a great impact on infant brain development. The pioneer study in this field was conducted by Dr. Meaney's group at McGill University. They found that rat pups that received intensive tactile stimulation, such as licking and grooming from their mother, showed lower stress responses during their adulthood (Liu et al., 1997; Meaney, 2001). Pups raised by higher licking and grooming mothers had a higher level of glucocorticoid receptor expression in the hippocampus, and showed an enhanced negative feedback ability along the hypothalamus-pituitary-adrenal axis of stress responses. Such tactile stimulation releases $5 \mathrm{HT}$ in the hippocampus of pups, which epigenetically modulates the CpG islands of glucocorticoid receptors (Weaver et al., 2004a,b).
These effects of tactile stimulation are most important just after birth, because the epigenetic changes of DNA methylation are determined within the first three days of the neonatal period. However, the mother-infant relationship during the later neonatal period also produces a long-lasting modulation of infant behavior, implying that not only the tactile stimulation, but other social cues from mother to infant have a significant role on the development of pups (Kikusui and Mori, 2009; Curley et al., 2010; Mogi et al., 2011).

Although the specific cues from mother to infant have not yet been identified, the most well studied model is a maternal deprivation paradigm. When mutually strongly bonded mothers and young are separated prematurely, bouts of reinstatement behavior such as locomotion (searching) and distress vocals are exhibited by both the mother and the young, interspersed with periods of energy-conserving depression in most mammals (Panksepp, 2004), indicating the importance of motherinfant bonding across species. Bowlby referred to these phases as "protest" and "despair," respectively (Bowlby, 1969). The impact of deprivation of mother-infant bonding is not temporary, and the effects can be observed much later in life. In rat and mouse models, maternally deprived animals show higher anxiety and stress responses, as well as lower learning and memory abilities in their adulthood (Levine, 1967; Plotsky and Meaney, 1993; Plotsky et al., 1993; Liu et al., 2000), suggesting that the epigenetic modulation of the brain function by maternal deprivation has long-lasting effects. We have studied the effects of early weaning, where the animals are weaned one week earlier than normal, and found that early weaned mice and rats showed higher levels of anxiety-related behaviors, as well as higher stress responses in their adulthood, even though no growth retardation was observed (Kikusui et al., 2004; Kikusui and Mori, 2009; Mogi et al., 2011). These results show that not only the early neonatal period, but also the mother-infant relationship in the later lactating period, has a profound impact on brain development in the infant.

When early-weaned male mice are grouped together, high levels of agonistic behavior, accompanied by an elevation in glucocorticoid levels were found (Nakamura et al., 2008). In young male elephants, following the loss of their mothers and other family members due to poaching, unusually violent behavior has been attributed to the termination of social interaction with older elephants, including parents, and the loss of behavioral inhibition that would normally result from the presence of older males (Slotow et al., 2000). The critical role of older males in normal social development was clearly shown when researchers re-introduced older bulls to quell the young males' violence, and hyperaggression and abnormally early musth cycles (periods of sexual activity and hormonal shifts) both ceased. Scott and Fuller (1965) studied behavioral development in dogs, and found that the socialization period, during which puppies stop suckling but continue to live with their parents, was crucial to their behavioral and social development. The social cues from mother or adult to juvenile animals responsible for these phenomena has not been determined, and we should consider the influence of social environment on the development of normal brain function in juvenile animals. 


\section{NEUROENDOCRINE REGULATION OF MOTHER-PUP INTERACTIONS}

Female rats or mice that have never given birth usually withdraw from or avoid pups, and it has been intensively studied how neuroendocrine changes at parturition act to enhance the neural mechanisms responsible for maternal behavior, such as those occurring in the mPOA of the hypothalamus (Numan and Stolzenberg, 2009). A great deal of evidence suggests that the oxytocin neuroendocrine system plays a key role in the initiation of maternal behavior following birth. It is now clear that oxytocin, which is synthesized in the neurons of the hypothalamus (specifically in the paraventricular nucleus $[\mathrm{PVN}]$ and supraoptic nucleus), is released not only into the general circulation from the posterior pituitary, but centrally during vaginal stimulation and parturition, to act upon oxytocin receptors widely expressed throughout the central nervous system (Forsling, 1986; Barberis and Tribollet, 1996; Gimpl and Fahrenholz, 2001). Moreover, maternal behavior was found to be greatly impaired in postparturient rats that received PVN lesions during pregnancy (Insel and Harbaugh, 1989), and in female rats administrated an oxytocin antagonist immediately after parturition (van Leengoed et al., 1987). Furthermore, oxytocin receptors proliferate in many forebrain areas including the $\mathrm{mPOA}$, ventromedial hypothalamus, and bed nucleus of the stria terminalis of female rats at the time of partition (Jirikowski et al., 1989; Pedersen et al., 1994). These alterations in oxytocin receptor expression may be partially responsible for the induction of maternal behavior, since intraventricular and $\mathrm{mPOA}$ infusion of oxytocin induces a rapid onset of maternal behavior in virgin female rats (Pedersen and Prange Jr, 1979; Pedersen et al., 1994).

In rodent mothers, the maintenance of maternal behavior is dependent on stimuli from the pups. If new mothers are separated from their pups and not permitted to interact with them, their maternal responsiveness declines over the first postpartum week (Orpen and Fleming, 1987). Among the various pup stimuli, physical contact is thought to play an important role in the maintenance of maternal behavior. Suckling of the nipple is a representative stimulus in lactating females, because suckling is known to stimulate oxytocin release not only into the peripheral circulation to cause milk ejection, but also into the brain (Neumann et al., 1993). Several types of tactile stimuli such as touch or massage-like stroking and warmth have also been shown to stimulate oxytocin release in rats; however, these are not associated with lactation physiology (Uvnas-Moberg et al., 1993; Agren et al., 1995).

Although exteroceptive social cues from pups, such as infant's odors or vocalizations, may not have as strong an effect on the development of nurturance as physical stimuli (Stern, 1983), they do play an important role in making mothers seek their pups out and maintain proximity. The infant's odors are used as not only an attractive signal to mothers, as described in previous sections, but also for the mother's recognition of their own infants (Ostermeyer and Elwood, 1983). As described above for rats, infant's odors have been shown to be quite aversive to females before parturition and lactation, whereas on the boundary of parturition, these odors become very potent attractive stimuli (Levy et al., 2004). This change in the mother's responsiveness is governed by hormonal changes at parturition, and it is suggested that the oxytocin system participates primarily in this process. Pregnancy and parturition enhance the sensitivity and neural firing in the olfactory bulb in rats, and oxytocin infusion directly into the olfactory bulb increases the firing frequency and spontaneous excitatory postsynaptic currents in granule cells (Yu et al., 1996) via both pre- and postsynaptic mechanisms, a process integral to olfactory recognition memory (Engelmann et al., 1998; Dluzen et al., 2000; Osako et al., 2001). Similar to the responsiveness to pup odors, the response to pup USVs has also been shown to be far more pronounced in mothers than in naïve females (Koch and Ehret, 1989; Okabe et al., 2010); however, the neuroendocrine mechanisms responsible for this change in response to pup USVs has not been elucidated.

Oxytocin-induced maternal behavior is reinforced by infants' innate attachment behaviors, i.e., vocalization or milk suckling for sustenance. Interestingly, it has been suggested that the oxytocin neuroendocrine system is also involved in these behaviors in infants. Central administration of oxytocin has been found to reduce the frequency of isolation-induced USV in young rat pups (Insel and Winslow, 1991), suggesting a possible role for central oxytocin in the affective "calm" response infants display during social contact. Central oxytocin also induces paw sucking in neonatal rats (Nelson and Alberts, 1997). These innate attachments regulated by oxytocin ensure the infant's physical development, and the subsequent development of adequate attachment behaviors accompanied by growth of the pup. In return, adequate attachments maintain maternal behaviors in response to pup stimuli.

\section{SOCIAL EXPERIENCE MODULATING THE MOTHER-PUP INTERACTION}

Maternal behavior is regulated by genetic and epigenetic backgrounds. Regarding epigenetic modulations of maternal behavior, "non-genomic transmission" has been indicated in a rat model (Champagne et al., 2001; Champagne and Meaney, 2001; Weaver et al., 2004a). As described above, pups that received higher levels and more intensive care from their mother in turn showed higher licking and grooming behavior toward their own offspring after delivery (Francis et al., 1999a,b). This behavioral transmission was persistent even when the pups were cross-fostered to other mother rats; that is, when pups born to the lower licking and grooming mother were cross-fostered to the higher licking and grooming mother, they showed higher maternal care to their own pups (Francis et al., 1999a,b). This transmission was clearly demonstrated by the epigenetic modification of the estrogen receptor genes in the MPOA in the hypothalamus (Champagne et al., 2001; Champagne and Meaney, 2001). The female rats that received higher licking and grooming behavior from the mother showed higher estrogen receptor expression in the same cells that also express oxytocin receptor. Estrogen receptor activation acts to induce oxytocin receptor expression, therefore, higher estrogen receptor expression results in higher oxytocin receptor expression. The oxytocin system in the mPOA is associated with the induction of maternal behavior (Pedersen et al., 1994). Therefore, these epigenetic modulations are plausible candidates to cause the individual differences in maternal behavior. 
Early-weaned females also showed lower maternal behavior when they became mothers (Kikusui et al., 2005). This may be because the early-weaned females were deprived maternal care in the third week of the postnatal period, resulting in lower maternal behavior in their adulthood. Although the responsible social cues from mother to infant mice in this model was not identified, it is suggested that not only the early neonatal period, but also mother-infant interactions during the late lactating period can epigenetically modulate the maternal behavior. Another interesting model to aid our understanding of the significance of social experience in the late neonatal period is the alloparental care experience in juvenile animals. For example, if the juveniles have a chance to show maternal or paternal-like behavior toward their younger siblings (termed alloparental care), they will show higher levels of nursing behavior toward their own offspring later in life (Riedman, 1982). These phenomena are widely observed in mammals, but detailed analyses have focused mainly on primates (Quiatt, 1979). In humans, similar mechanisms have been suggested, but not clearly defined (Hrdy, 1999).

Social experience affects not only neonates or juveniles, but also adult rodents in terms of maternal behavior. Unlike lactating mothers, most sexually and parentally naïve adult rodents avoid physical contact with pups when they are first introduced to them (Terkel and Rosenblatt, 1971). Particularly noticeable in rats, [although juvenile and premature rats (18-42 days old) will approach pups and initiate physical contact with unfamiliar pups], inexperienced adult rats show clear avoidance or attacking behavior toward unfamiliar pups (Terkel and Rosenblatt, 1971). However, after 5-8 days of continuous cohabitation with donor pups, they first become tolerant of proximate contact with pups, and then start caring for the pups, which includes licking, retrieving, crouching, and nest building (Rosenblatt, 1967). This cohabitation effect is termed "pup sensitization." Once becoming fully parental by pup sensitization, virgin female rats can maintain a high level of parental responsiveness for several weeks after separation from pups (Bridges and Scanlan, 2005).

In mice, virgin females are often said to be "spontaneously parental" like postpartum mothers, because a cohabitation period of only $30 \mathrm{~min}$ can induce parental care behavior (Gandelman, 1972). At the first time of exposure to the pups, female mice sniff the pups from a distance with their eyes closed, and then investigate the pups. During these bouts of investigation, the virgin female runs between the pup and the nest, apparently showing an approach-avoidance conflict. In addition, their retrieving latency decreases with repetition of the presentation of donor pups, indicating that previous parenting experience enhances parental behavior (Ehret et al., 1987). Once they become parental through pup sensitization, they seldom revert to rejecting pups, at least during successive daily pup exposure. Therefore, parental experience can develop the parental responsiveness in a long-lasting manner in rodents.

Reproductive experiences such as mating, pregnancy, and parturition are also important factors for expression of maternal behavior. Graber showed that even uterine distention can shorten the onset latency of maternal behavior in rats (Graber and Kristal, 1977). Similar to Graber's study, in our previous study, mating experience facilitates maternal behavior in female mice. However, our results also showed that the additional experience of parenting is necessary for the complete induction of maternal behavior in female mice (Okabe et al., 2010).

These social experiences are all accompanied by the release of oxytocin, not only from the posterior pituitary, but also through central release in order to act upon oxytocin receptors widely expressed throughout several brain regions. Interestingly, increases in oxytocin levels have been measured both centrally and peripherally following genital and cervical stimulation in several species including rodents and ruminants (Kendrick and Keverne, 1992; Sansone et al., 2002). In addition, tactile or nipple suckling stimuli can stimulate the release of oxytocin (UvnasMoberg et al., 1993; Agren et al., 1995), which is similar physical contact to that experienced during pup sensitization. Therefore, it is possible that pup sensitization as well as sexual experience could facilitate maternal behavior through activation of the oxytocin system in the brain, particularly the MPOA in the hypothalamus.

\section{IMPLICATIONS FOR HUMAN MOTHER-INFANT INTERACTIONS}

In experimental animals, it has been shown that oxytocin strongly influences maternal care, and the prosocial effects of oxytocin have been widely recognized (Francis et al., 2000; Williams et al., 2001; Caldwell and Young, 2006). However, the neuroendocrinological research of mother-infant relations in humans is now being progressively undertaken, although it remains unclear how oxytocin links mother-infant relationship and post-growth offspring. In this section, we review the current research on the effects of oxytocin in mother-infant relationships in humans.

Human research on the role of oxytocin in mother-infant relations is divided into two types. One type involved the investigation of the association between the mother's oxytocin levels and maternal behavior or degree of attachment, and the other type involved examining the influence of parental care experienced by the infants on their oxytocin levels. In the first type, maternal oxytocinergic status from pregnancy to early postnatal was evaluated. Levine et al. (2007) assayed plasma oxytocin levels in the first and third trimesters of pregnancy and the first postpartum month, and the subjects completed a psychological scale (Maternal-Fetal Attachment Scale; MFAS) in the third trimester. Although there was no significant correlation between oxytocin levels during pregnancy and MFAS scores, the group having increasing oxytocin patterns during pregnancy tended to show higher MFAS scores. Feldman et al. (2007) examined maternal plasma oxytocin levels at the same points as those used in Levine's study along with analysis of maternal representations and maternal behaviors in the first postpartum month. Oxytocin levels in early pregnancy and postpartum correlated significantly with attachment representation and maternal behaviors, such as gaze at infants, affectionate touch, and frequent infant checking. Feldman and colleagues also focused on mother-infant behavioral synchrony and showed a correlation between maternal salivary and plasma oxytocin levels and affection synchrony (Feldman et al., 2010, 2011). Strathearn et al. (2009) conducted an interview with pregnant women and divided them according to their attachment style; that is, mothers with a secure attachment style and those with an insecure-avoidant attachment style. At seven months after 
birth, they assayed maternal serum oxytocin within a short time of mother-infant interaction, and found that oxytocin levels were increased significantly in the secure mother group. Gordon et al. (2010) examined both maternal and paternal plasma oxytocin levels after a period of interaction with their infants, and found that maternal oxytocin levels correlated with affectionate parenting behavior and paternal levels correlated with stimulatory parenting behavior. These results suggest the presence of a clear positive relationship between mothering style and attachment, and oxytocin activity in mothers.

Conversely, Feldman et al. (2010) examined infants' salivary oxytocin levels following parent-infant interaction, and found that parent and infant oxytocin levels were significant correlated both pre-interaction and post-interaction. Moreover, under conditions of high levels of affect behavioral synchrony in the parent-infant interaction, infants whose parents had high oxytocin levels had significantly higher oxytocin level than infants whose parents had relatively low oxytocin levels. A recent study showed that social vocalization from the mother to the daughter stimulates oxytocin release and reduces stress responses in humans (Seltzer et al., 2010). These results suggest that social signals from the mother stimulate the oxytocin system in infants.

However, Fries et al. (2005) elucidated a relationship between infant's oxytocin levels and early negative experiences. They measured urinary oxytocin levels of children aged approximately 4 years who had experienced early neglect and institutionalization. Although the children were adopted into new families, their oxytocin levels tended to be lower than in children who were reared by their biological parents. Moreover, Gordon et al. (2008) reported that plasma oxytocin levels of young adults had a significant correlation with their maternal and paternal care, which was measured by self-report (Parental Bonding Instrument). Meinlschmidt and Heim (2007) targeted young adults who had experienced early childhood trauma by divorce or separation of parents at an age of less than 13 years. They measured salivary cortisol levels after the nasal administration of either oxytocin or placebo. In the control group, oxytocin administration decreased cortisol levels; however, this was not the case in the divorce/separation experienced group. Heim et al. (2009) examined the effects of early-life adversity on oxytocin activity in the central nervous system in adult women. Oxytocin concentrations in cerebrospinal fluid (CSF) in maltreated women decreased as compared to healthy controls. These studies suggested that quality of parental care during the juvenile period might influence oxytocinergic function over a longer duration, until adulthood.

In humans, the behavior of oxytocin can be measured mainly in the periphery, such as the plasma, saliva, and urine (Amico et al., 1987; Carter et al., 2007). Moreover, human interactions are extremely complex and encompass various individual differences. These factors may prove to be an obstacle for elucidating the precise influences of oxytocin in humans. On the other hand, research on humans can make use of psychological introspective methods that allow to map out behavior and feelings in more detail. Moreover, recent fMRI research has begun to investigate the relationship between oxytocin-related areas in the central nervous system and social stimuli involved in bonding (Strathearn et al., 2009; Riem et al., 2011). The research in humans may be able to provide a new viewpoint on the role of oxytocin in mother-infant relationships using a combination of endocrinological, behavioral, and psychological methods.

The comparison of rodent and human studies is valuable for translational research in this field. In the rodent models, the release of oxytocin in the amygdala was increased by social olfactory cues in mice (Ferguson et al., 2001), and oxytocin administration to lactating mother rats activated the brain area including the olfactory system and amygdala, as detected by fMRI (Febo et al., 2005). Therefore, the oxytocin system is mainly acting on the olfactory circuit. The release of oxytocin in the amygdala, which is the higher brain area of olfactory circuit, also enhances the effect on dopamine transmission in the nucleus accumbens, which is essential for social bonding (Broad et al., 2006). On the other hand, in monkeys and humans, it was found that social visual cues, such as gazing, activated the oxytocin systems. Thus, the sensory inputs that stimulate oxytocin in the brain are different in rodents and primates. Interestingly, visual cues in monkeys activate neurons in the amygdala (Morris et al., 1996; Gothard et al., 2007), implying that the amygdala is the key region for the social signal circuit. There is a possibility that similar mechanisms may underlie bonding in both humans and rodents, nevertheless the nature of the cues that can stimulate oxytocin has shifted over the course of evolution (Broad et al., 2006).

\section{CONCLUSION}

We have presently described the mutual communication between mother and infants, and discussed the manner in which this type of communication creates a fundamental bonding between mother and infant. Although the social cues used by human beings are not so clearly defined, vocal and visual stimuli from both mother and infants to others seem important. Moreover, tactile stimulation between mother and infants is a basic communicative tool that is used to bond with each other. Further studies are required to clarify these issues in humans. The summarized role of the oxytocin system in reciprocal communication is shown in Figure 1.

The oxytocin system may play a pivotal role in bonding formation. In animal models, it has been clearly shown that motherinfant bonding is mediated by the oxytocin systems, particularly in ewes (Kendrick, 2004). As described above, experiences of social and affiliative interactions, such as parenting and mating, stimulate the oxytocin system. The oxytocin then enhances their own parenting behavior toward their infants, indicating that there is a positive loop of oxytocin and parenting behavior in individuals. If the infants receive higher levels of parenting care, their oxytocin system is stimulated and makes them seek more contact with their parent. During parenting, animals show increased oxytocin related to infant attachment behavior. Thus, there is also a positive loop of attachment-parenting behavior via the oxytocin system on each side of infant-mother dyad. Moreover, if the infant receives higher parenting behavior, they will develop an enhanced oxytocin system and oxytocin-related behaviors in their adulthood, such as parenting and social affiliative behaviors. Therefore, there is also a positive loop of parenting/affiliative behavior that crosses generations. This idea is schematically represented in Figure 2. 


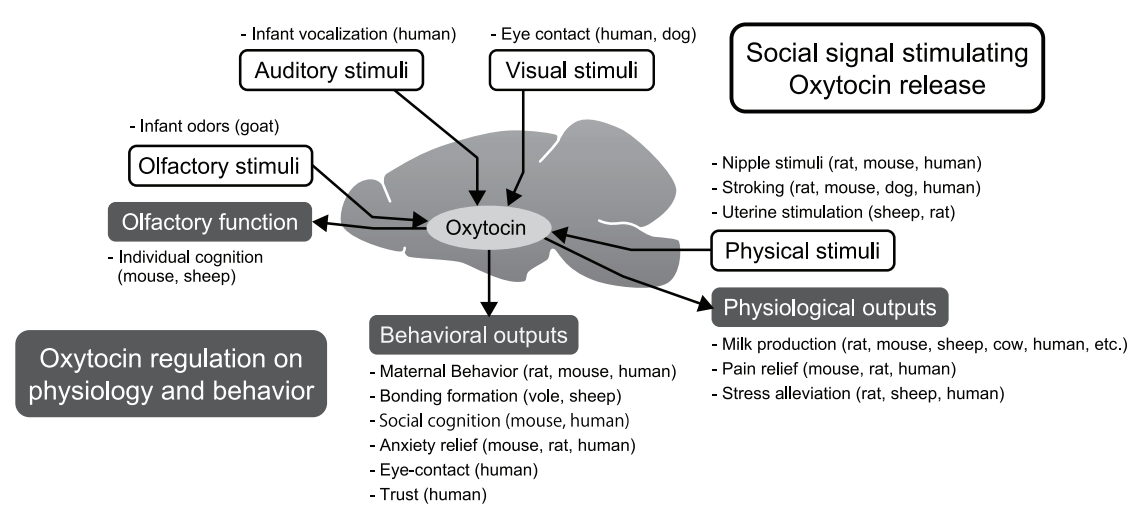

FIGURE 1 | Summary of the role of the oxytocin system in reciprocal communication. Central oxytocin release is stimulated by multiple sensory signals, such as olfactory, auditory, visual, and physical inputs. In particular, physiological stimuli are known to induce oxytocin system activation in mammals. When oxytocin release is increased in the central nervous system, many sensory, physiological, and behavioral functions are enhanced. Maternal as well as affiliative behaviors are enhanced by oxytocin. Additionally, negative responses, such as pain, stress endocrine, and anxiety behaviors are diminished by oxytocin.

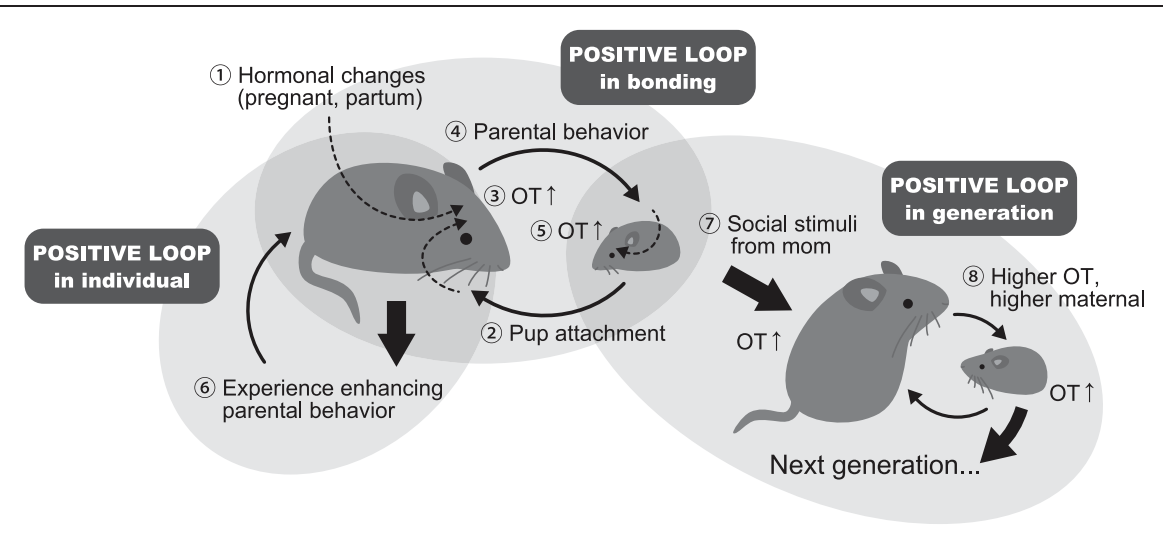

FIGURE 2 | A schematic illustrating the positive loop of social bonding controlled by oxytocin. The mother mouse becomes pregnant and then at partum, certain hormonal changes occur (1). After delivery, new-born infants show attachment behavior toward the mother (2). The hormonal changes related to partum and attachment behavior from the infant stimulate the release of centrally acting oxytocin (OT) (3). OT in the maternal brain facilitates parenting behavior toward the pups (4). This parenting behavior also stimulates infant OT release in the brain (5). Therefore, there is a clear positive loop of OT release in the mother-infant dyad. Once the mother experiences parenting, her maternal behavior is persistently maintained (6) positive loop in individuals. Intensive parenting care stimulates the infants' brain development (7), which in turn brings about higher OT and parenting activities in their adulthood (8). This intensive care is also non-genetically transmitted to the next generation (positive loop in the generation).
Oxytocin is of vital importance for the induction of maternal behavior as well as for social cognition (Young and Wang, 2004). Dissociating the role of oxytocin in bond formation and in social cognition is likely to be difficult, but it is plausible that oxytocin may act as the master mediator in recognizing the object of the bonding-partner and connecting to each other. This classic neuropeptide, seen in a wide variety of vertebrate species including humans, has a fascinating character, which may be vital to understand the biological significance of bonding, from evolutional and ethological points of view (Young and Wang, 2004).

Despite the difficulties involved in measuring oxytocin activity in the human brain, as well as standardizing environments and experiences, and considering genetic diversity among humans, there have been some fascinating results obtained in the field of mother-infant relationship in humans. We have recently found that human-dog interaction stimulates oxytocin release in humans, with this being particularly related to the dog's initiation of gaze communications (Nagasawa et al., 2009). This type of study will shed further light on the nature of the biological bonding system in humans, including inter-species bonding like human-dog connections. We are only at the starting point of the journey to reveal neuronal mechanisms involved in human cognition and bonding formation.

\section{ACKNOWLEDGMENTS}

This work was financially supported by Grants-in-Aid for Scientific Research from the Ministry of Education, Culture, Sports, Science, and Technology of Japan (\#14760187, \#23248049 \#23132510 to Takefumi Kikusui and \#23780301 to Kazutaka Mogi) and Promotion and Mutual Aid Corporation for Private Schools of Japan, Grant-in-Aid for Matching Fund Subsidy for Private Universities. 


\section{REFERENCES}

Agid, O., Shapira, B., Zislin, J., Ritsner, M., Hanin, B., Murad, H., Troudart, T., Bloch, M., Heresco-Levy, U., and Lerer, B. (1999). Environment and vulnerability to major psychiatric illness: a case control study of early parental loss in major depression, bipolar disorder and schizophrenia. Mol. Psychiatry 4, 163-172.

Agren, G., Lundeberg, T., UvnasMoberg, K., and Sato, A. (1995). The oxytocin antagonist 1deamino-2-D-Tyr-(Oet)-4-Thr-8Orn-oxytocin reverses the increase in the withdrawal response latency to thermal, but not mechanical nociceptive stimuli following oxytocin administration or massage-like stroking in rats. Neurosci. Lett. 187, 49-52.

Amico, J. A., Ulbrecht, J. S., and Robinson, A. G. (1987). Clearance studies of oxytocin in humans using radioimmunoassay measurements of the hormone in plasma and urine. J. Clin. Endocrinol. Metab. 64, 340-345.

Barberis, C., and Tribollet, E. (1996). Vasopressin and oxytocin receptors in the central nervous system. Crit. Rev. Neurobiol. 10, 119-154.

Benuck, I., and Rowe, F. A. (1975). Centrally and peripherally induced anosmia: influences on maternal behavior in lactating female rats. Physiol. Behav. 14, 439-447.

Bowlby, J. (1969). Attachment, Vol. 1 of Attachment and Loss. London: Pimlico. New York: Reprint of Hogarth.

Branchi, I., Santucci, D., and Alleva, E. (2001). Ultrasonic vocalisation emitted by infant rodents: a tool for assessment of neurobehavioural development. Behav. Brain Res. 125, 49-56.

Bridges, R. S., and Scanlan, V. F. (2005). Maternal memory in adult, nulliparous rats: effects of testing interval on the retention of maternal behavior. Dev. Psychobiol. 46, 13-18.

Broad, K. D., Curley, J. P., and Keverne, E. B. (2006). Mother-infant bonding and the evolution of mammalian social relationships. Philos. Trans. R. Soc. Lond. B. Biol. Sci. 361, 2199-2214.

Brouette-Lahlou, I., Vernet-Maury, E., and Chanel, J. (1991). Is rat-dam licking behavior regulated by pups' preputial gland secretion? Learn. Behav. 19, 177-184.

Brown, A. M. (1973). High levels of responsiveness from the inferior colliculus of rodents at ultrasonic frequencies. J. Comp. Physiol A: Neuroethol. Sens. Neural Behav. Physiol. 83, 393-406.
Caldwell, H., and Young, W. (2006). "Oxytocin and Vasopressin: genetics and behavioral implications," in Structure, ed R. Lim (New York, NY: Springer), 573-607.

Carter, C., Pournajafi-Nuzarloo, H., and Kramer, K. M. (2007). Oxytocin: behavioral associations and potential as a salivary biomarker. Ann. N.Y. Acad. Sci. 1098, 312-322.

Champagne, F., Diorio, J., Sharma, S. and Meaney, M. J. (2001). Naturally occurring variations in maternal behavior in the rat are associated with differences in estrogeninducible central oxytocin receptors. Proc. Natl. Acad. Sci. U.S.A. 98, 12736-12741.

Champagne, F., and Meaney, M. J. (2001). Like mother, like daughter: evidence for non-genomic transmission of parental behavior and stress responsivity. Prog. Brain Res. 133, 287-302.

Cohen, L., Rothschild, G., and Mizrahi, A. (2011). Multisensory integration of natural odors and sounds in the auditory cortex. Neuron 72, 357-369.

Curley, J. P., Rock, V., Moynihan, A. M., Bateson, P., Keverne, E. B., and Champagne, F. A. (2010). Developmental shifts in the behavioral phenotypes of inbred mice: the role of postnatal and juvenile social experiences. Behav. Genet. 40, 220-232.

DeVries, A. C. (2002). Interaction among social environment, the hypothalamic-pituitary-adrenal axis, and behavior. Horm. Behav. 41, 405-413.

Dluzen, D. E., Muraoka, S., Engelmann, M., Ebner, K., and Landgraf, R. (2000). Oxytocin induces preservation of social recognition in male rats by activating alphaadrenoceptors of the olfactory bulb. Eur. J. Neurosci. 12, 760-766.

Ehret, G. (2005). Infant rodent ultrasounds? A gate to the understanding of sound communication. Behav. Genet. 35, 19-29.

Ehret, G., Koch, M., Haack, B., and Markl, H. (1987). Sex and parental experience determine the onset of an instinctive behavior in mice. Naturwissenschaften 74, 47.

Elwood, R. W., and Keeling, F. (1982). Temporal organization of ultrasonic vocalizations in infant mice. Dev. Psychobiol. 15, 221-227.

Engelmann, M., Ebner, K., Wotjak, C. T., and Landgraf, R. (1998). Endogenous oxytocin is involved in short-term olfactory memory in female rats. Behav. Brain Res. 90, 89-94.
Fang, L. Y., Quan, R. D., and Kaba, H. (2008). Oxytocin facilitates the induction of long-term potentiation in the accessory olfactory bulb. Neurosci. Lett. 438, 133-137.

Farrell, W. J., and Alberts, J. R. (2002). Stimulus control of maternal responsiveness to Norway rat (Rattus norvegicus) pup ultrasonic vocalizations. J. Comp. Psychol. 116, 297-307.

Febo, M., Numan, M., and Ferris, C. F. (2005). Functional magnetic resonance imaging shows oxytocin activates brain regions associated with mother-pup bonding during suckling. J. Neurosci. 25, 11637-11644.

Feldman, R., Gordon, I., and Zagoory-Sharon, O. (2010). The cross-generation transmission of oxytocin in humans. Horm. Behav. 58, 669-676.

Feldman, R., Gordon, I., and ZagoorySharon, O. (2011). Maternal and paternal plasma, salivary, and urinary oxytocin and parent-infant synchrony: considering stress and affiliation components of human bonding. Dev. Sci. 14, 752-761.

Feldman, R., Weller, A., ZagoorySharon, O., and Levine, A. (2007). Evidence for a neuroendocrinological foundation of human affiliation: plasma oxytocin levels across pregnancy and the postpartum period predict mother-infant bonding. Psychol. Sci. 18, 965-970.

Ferguson, J. N., Aldag, J. M., Insel, T. R. and Young, L. J. (2001). Oxytocin in the medial amygdala is essential for social recognition in the mouse. $J$. Neurosci. 21, 8278-8285.

Fleming, A. S., Cheung, U., Myhal, N., and Kessler, Z. (1989). Effects of maternal hormones on "timidity" and attraction to pup-related odors in female rats. Physiol. Behav. 46, 449-453.

Forsling, M. (1986). Regulation of oxytocin release. Curr. Topics Neuroendocrinol. 6, 19-53.

Francis, D. D., Diorio, J., Liu, D., and Meaney, M. J. (1999a). Nongenomic transmission across generations of maternal behavior and stress responses in the rat. Science 286, 1155.

Francis, D. D., Champagne, F. A., Liu, D., and Meaney, M. J. (1999b). Maternal care, gene expression, and the development of individual differences in stress reactivity. Ann. N.Y. Acad. Sci. 896, 66-84.

Francis, D. D., Champagne, F. A., and Meaney, M. J. (2000). Variations in maternal behaviour are associated with differences in oxytocin receptor levels in the rat. $J$. Neuroendocrinol. 12, 1145-1148.
Fries, A. B., Ziegler, T. E., Kurian, J. R., Jacoris, S., and Pollak, S. D. (2005). Early experience in humans is associated with changes in neuropeptides critical for regulating social behavior. Proc. Natl. Acad. Sci. U.S.A. 102, 17237-17240.

Gandelman, R. (1972). Induction of pup killing in female mice by androgenization. Physiol. Behav. 9, 101-102.

Gimpl, G., and Fahrenholz, F. (2001). The oxytocin receptor system: structure, function, and regulation. Physiol. Rev. 81, 629-683.

Gordon, I., Zagoory-Sharon, O., Leckman, J. F., and Feldman, R. (2010). Oxytocin and the development of parenting in humans. Biol. Psychiatry 68, 377-382.

Gordon, I., Zagoory-Sharon, O., Schneiderman, I., Leckman, J. F., Weller, A., and Feldman, R. (2008) Oxytocin and cortisol in romantically unattached young adults: associations with bonding and psychological distress. Psychophysiology 45, 349-352.

Gothard, K. M., Battaglia, F. P., Erickson, C. A., Spitler, K. M., and Amaral, D. G. (2007). Neural responses to facial expression and face identity in the monkey amygdala. J. Neurophysiol. 97, 1671-1683.

Graber, G. C., and Kristal, M. B. (1977). Uterine distention facilitates the onset of maternal behavior in pseudopregnant but not in cycling rats. Physiol. Behav. 19, 133-137.

Heim, C., and Nemeroff, C. B. U. (2001). The role of childhood trauma in the neurobiology of mood and anxiety disorders: preclinical and clinical studies. Biol. Psychiatry 49, 1023-1039.

Heim, C., Young, L. J., Newport, D. J., Mletzko, T., Miller, A. H., and Nemeroff, C. B. (2009). Lower CSF oxytocin concentrations in women with a history of childhood abuse. Mol. Psychiatry 14, 954-958.

Hennessy, M. B. (1997). Hypothalamicpituitary-adrenal responses to brief social separation. Neurosci. Biobehav. Rev. 21, 11-29.

Hildyard, K. L., and Wolfe, D. A. (2002). Child neglect: developmental issues and outcomes. Child Abuse Negl. 26, 679-695.

Hrdy, S. B. (1999). Mother Nature: A History of Mothers, Infants, and Natural Selection. New York, NY: Pantheon Books.

Insel, T. R., and Harbaugh, C. R. (1989). Lesions of the hypothalamic paraventricular nucleus disrupt the initiation of maternal behavior Physiol. Behav. 45, 1033-1041. 
Insel, T. R., and Winslow, J. T. (1991). Central administration of oxytocin modulates the infant rat's response to social isolation. Eur. J. Pharmacol. 203, 149-152.

Jirik-Babb, P., Manaker, S., Marie Tucker, A., and Hofer, M. A. (1984). The role of the accessory and main olfactory systems in maternal behavior of the primiparous rat. Behav. Neural Biol. 40, 170-178.

Jirikowski, G. F., Caldwell, J. D., Pilgrim, C., Stumpf, W. E., and Pedersen, C. A. (1989). Changes in immunostaining for oxytocin in the forebrain of the female rat during late pregnancy, parturition and early lactation. Cell Tissue Res. 256, 411-417.

Kendrick, K. M. (2000). Oxytocin, motherhood and bonding. Exp. Physiol. 85(Suppl.) 111S-124S.

Kendrick, K. M. (2004). The neurobiology of social bonds. J. Neuroendocrinol. 16, 1007-1008.

Kendrick, K., and Keverne, E. (1992). Control of synthesis and release of oxytocin in the sheep brain. Ann. N.Y. Acad. Sci. 652, 102-121.

Kikusui, T., and Mori, Y. (2009). Behavioural and neurochemical consequences of early weaning in rodents. J. Neuroendocrinol. 21, 427-431.

Kikusui, T., Isaka, Y., and Mori, Y. (2005). Early weaning deprives mouse pups of maternal care and decreases their maternal behavior in adulthood. Behav. Brain Res. 162, 200-206.

Kikusui, T., Takeuchi, Y., and Mori, Y. (2004). Early weaning induces anxiety and aggression in mice. Physiol. Behav. 81, 37-42.

Kikusui, T., Winslow, J. T., and Mori, Y. (2006). Social buffering: relief from stress and anxiety. Philos. Trans. R. Soc. Lond. B. Biol. Sci. 361, 2215-2228.

Kinsley, C. H., and Bridges, R. S. (1990). Morphine treatment and reproductive condition alter olfactory preferences for pup and adult male odors in female rats. Dev. Psychobiol. 23, 331-347.

Koch, M., and Ehret, G. (1989). Estradiol and parental experience, but not prolactin are necessary for ultrasound recognition and pupretrieving in the mouse. Physiol. Behav. 45, 771-776.

Kolunie, J. M., and Stern, J. M. (1995). Maternal aggression in rats: effects of olfactory bulbectomy, ZnSO4induced anosmia, and vomeronasal organ removal. Horm. Behav. 29, 492-518.

Levine, A., Zagoory-Sharon, O., Feldman, R., and Weller, A.
(2007). Oxytocin during pregnancy and early postpartum: individual patterns and maternal-fetal attachment. Peptides 28, 1162-1169.

Levine, S. (1967). Maternal and environmental influences on the adrenocortical response to stress in weanling rats. Science 156, 258-260.

Levy, F., Keller, M., and Poindron, P. (2004). Olfactory regulation of maternal behavior in mammals. Horm. Behav. 46, 284-302.

Liu, D., Diorio, J., Day, J. C., Francis, D. D., and Meaney, M. J. (2000) Maternal care, hippocampal synaptogenesis and cognitive development in rats. Nat. Neurosci. 3, 799-806.

Liu, D., Diorio, J., Tannenbaum, B., Caldji, C., Francis, D., Freedman, A., Sharma, S., Pearson, D., Plotsky, P. M., and Meaney, M. J. (1997). Maternal care, hippocampal glucocorticoid receptors, and hypothalamic-pituitary-adrenal responses to stress. Science 277, 1659-1662.

Liu, R. C., and Schreiner, C. E. (2007). Auditory cortical detection and discrimination correlates with communicative significance. PLoS Biol. 5, e173. doi: 10.1371/journal.pbio. 0050173

Meaney, M. J. (2001). Maternal care, gene expression, and the transmission of individual differences in stress reactivity across generations. Annu. Rev. Neurosci. 24, 1161-1192.

Meinlschmidt, G., and Heim, C. (2007). Sensitivity to intranasal oxytocin in adult men with early parental separation. Biol. Psychiatry 61, 1109-1111.

Mogi, K., Nagasawa, M., and Kikusui, T. (2011). Developmental consequences and biological significance of mother-infant bonding. Prog. Neuropsychopharmacol. Biol. Psychiatry 35, 1232-1241.

Morgan, H. D., Fleming, A. S., and Stern, J. M. (1992). Somatosensory control of the onset and retention of maternal responsiveness in primiparous Sprague-Dawley rats. Physiol. Behav. 51, 549-555.

Moriceau, S., and Sullivan, R. M. (2004). Unique neural circuitry for neonatal olfactory learning. $J$. Neurosci. 24, 1182-1189.

Morris, J. S., Frith, C. D., Perrett, D. I., Rowland, D., Young, A. W., Calder, A. J., and Dolan, R. J. (1996). A differential neural response in the human amygdala to fearful and happy facial expressions. Nature 383, 812-815.

Nagasawa, M., Kikusui, T., Onaka, T., and Ohta, M. (2009). Dog's gaze at its owner increases owner's urinary oxytocin during social interaction. Horm. Behav. 55, 434-441.

Nakamura, K., Kikusui, T., Takeuchi, Y., and Mori, Y. (2008). Changes in social instigation-and food restriction-induced aggressive behaviors and hippocampal 5HT1B mRNA receptor expression in male mice from early weaning. Behav. Brain Res.187, 442-448.

Nelson, E., and Alberts, J. R. (1997). Oxytocin-induced paw sucking in infant rats. Ann. N.Y. Acad. Sci. 807, 543-545.

Neumann, I., Russell, J. A., and Landgraf, R. (1993). Oxytocin and vasopressin release within the supraoptic and paraventricular nuclei of pregnant, parturient and lactating rats: a microdialysis study. Neuroscience 53, 65-75.

Norcross, J. L., and Newman, J. D. (1999). Effects of separation and novelty on distress vocalizations and cortisol in the common marmoset (Callithrix jacchus). Am. J. Primatol. 47, 209-222.

Nowak, R., Keller, M., and Levy, F. (2011). Mother-young relationships in sheep: a model for a multidisciplinary approach of the study of attachment in mammals. J. Neuroendocrinol. 23, 1042-1053.

Nowak, R., Porter, R. H., Levy, F., Orgeur, P., and Schaal, B. (2000). Role of mother-young interactions in the survival of offspring in domestic mammals. Rev. Reprod. 5, 153-163.

Numan, M. (2007). Motivational systems and the neural circuitry of maternal behavior in the rat. Dev. Psychobiol. 49, 12-21.

Numan, M., and Stolzenberg, D. S. (2009). Medial preoptic area interactions with dopamine neural systems in the control of the onset and maintenance of maternal behavior in rats. Front. Neuroendocrinol. 30 , 46-64.

Okabe, S., Nagasawa, M., Kihara, T., Kato, M., Harada, T., Koshida, N., Mogi, K., and Kikusui, T. (2010). The effects of social experience and gonadal hormones on retrieving behavior of mice and their responses to pup ultrasonic vocalizations. Zoolog. Sci. 27, 790-795.

Orpen, B. G., and Fleming, A. S. (1987). Experience with pups sustains maternal responding in postpartum rats. Physiol. Behav. 40, 47-54.

Osako, Y., Otsuka, T., Taniguchi, M., Oka, T., and Kaba, H. (2001). Oxytocin enhances presynaptic and postsynaptic glutamatergic transmission between rat olfactory bulb neurones in culture. Neurosci. Lett. 299, 65-68.

Ostermeyer, M. C., and Elwood, R. W. (1983). Pup recognition in Mus musculus: parental discrimination between their own and alien young. Dev. Psychobiol. 16, 75-82.

Panksepp, J. (2004). Affective Neuroscience: The Foundations of Human and Animal Emotions. USA: Oxford University Press.

Pedersen, C. A., Caldwell, J. D., Walker, C., Ayers, G., and Mason, G. A. (1994). Oxytocin activates the postpartum onset of rat maternal behavior in the ventral tegmental and medial preoptic areas. Behav. Neurosci. 108, 1163-1171.

Pedersen, C. A., and Prange Jr, A. J. (1979). Induction of maternal behavior in virgin rats after intracerebroventricular administration of oxytocin. Proc. Natl. Acad. Sci. U.S.A. 76, 6661-6665.

Plotsky, P. M., and Meaney, M. J. (1993). Early, postnatal experience alters hypothalamic corticotropinreleasing factor (CRF) mRNA, median eminence CRF content and stress-induced release in adult rats. Brain Res. Mol. Brain Res. 18, 195-200.

Plotsky, P. M., Thrivikraman, K. V., and Meaney, M. J. (1993). Central and feedback regulation of hypothalamic corticotropin-releasing factor secretion. Ciba Found. Symp. 172, 59-75.

Porter, R. H., and Winberg, J. (1999). Unique salience of maternal breast odors for newborn infants. Neurosci. Biobehav. Rev. 23, 439-449.

Quiatt, D. (1979). Aunts and mothers: adaptive implications of allomaternal behavior of nonhuman primates. Am. Anthropol. 81, 310-319.

Riedman, M. L. (1982). The evolution of alloparental care and adoption in mammals and birds. Q. Rev. Biol. 405-435.

Riem, M. M. E., BakermansKranenburg, M. J., Pieper, S., Tops, M., Boksem, M. A. S., Vermeiren, R. R. J. M., van Ijzendoorn, M. H., and Rombouts, S. A. R. B. (2011). Oxytocin modulates amygdala, insula, and inferior frontal gyrus responses to infant crying: a randomized controlled trial. Biol. Psychiatry 70, 291-297.

Rosenblatt, J. S. (1967). Nonhormonal basis of maternal behavior in the rat. Science 156, 1512-1514.

Ross, H. E., and Young, L. J. (2009). Oxytocin and the neural mechanisms regulating social cognition 
and affiliative behavior. Front. Neuroendocrinol. 30, 534-547.

Sansone, G. R., Gerdes, C. A., Steinman, J. L., Winslow, J. T., Ottenweller, J. E., Komisaruk, B. R., and Insel, T. R. (2002). Vaginocervical stimulation releases oxytocin within the spinal cord in rats. Neuroendocrinology 75, 306-315.

Scott, J., and Fuller, J. (1965). Genetics and Social Behavior in Dogs. Chicago, IL: The University Chicago Press.

Seegal, R. F., and Denenberg, V. H. (1974). Maternal experience prevents pup-killing in mice induced by peripheral anosmia. Physiol. Behav. 13, 339-341.

Seltzer, L. J., Ziegler, T. E., and Pollak, S. D. (2010). Social vocalizations can release oxytocin in humans. Proc. Biol. Sci. 277, 2661-2666.

Slotow, R., van Dyk, G., Poole, J., Page, B., and Klocke, A. (2000). Older bull elephants control young males. Nature 408, 425-426.

Stern, J. M. (1983). Maternal behavior priming in virgin and caesareandelivered Long-Evans rats: effects of brief contact or continuous exteroceptive pup stimulation. Physiol. Behav. 31, 757-763.

Strathearn, L., Fonagy, P., Amico, J., and Montague, P. R. (2009). Adult attachment predicts maternal brain and oxytocin response to infant cues. Neuropsychopharmacol. 34, 2655-2666.
Terkel, J., and Rosenblatt, J. S. (1971). Aspects of nonhormonal maternal behavior in the rat. Horm. Behav. 2, 161-171.

Uematsu, A., Kikusui, T., Kihara, T., Harada, T., Kato, M., Nakano, K., Murakami, O., Koshida, N., Takeuchi, Y., and Mori, Y. (2007). Maternal approaches to pup ultrasonic vocalizations produced by a nanocrystalline silicon thermoacoustic emitter. Brain Res. 1163, 91-99.

Uvnas-Moberg, K., Bruzelius, G., Alster, P., and Lundeberg, T. (1993). The antinociceptive effect of nonnoxious sensory stimulation is mediated partly through oxytocinergic mechanisms. Acta Physiol. Scand. 149, 199-204.

van Leengoed, E., Kerker, E., and Swanson, H. H. (1987). Inhibition of post-partum maternal behaviour in the rat by injecting an oxytocin antagonist into the cerebral ventricles. J. Endocrinol. 112, 275-282.

Wang, Z., and Aragona, B. J. (2004). Neurochemical regulation of pair bonding in male prairie voles. Physiol. Behav. 83, 319-328.

Wang, Z., and Storm, D. R. (2010). Maternal behavior is impaired in female mice lacking type 3 adenylyl cyclase. Neuropsychopharmacology 36, 772-781.

Weaver, I. C. G., Cervoni, N., Champagne, F. A., D'Alessio, A. C., Sharma, S., Seckl, J. R., Dymov,
S., Szyf, M., and Meaney, M. J. (2004a). Epigenetic programming by maternal behavior. Nat. Neurosci. 7, 847-854.

Weaver, I. C., Diorio, J., Seckl, J. R., Szyf, M., and Meaney, M. J. (2004b). Early environmental regulation of hippocampal glucocorticoid receptor gene expression: characterization of intracellular mediators and potential genomic target sites. Ann. N.Y. Acad. Sci. 1024, 182-212.

Williams, G. L., Gazal, O. S., Leshin, L. S., Stanko, R. L., and Anderson, L. L. (2001). Physiological regulation of maternal behavior in heifers: roles of genital stimulation, intracerebral oxytocin release, and ovarian steroids. Biol. Reprod. 65, 295-300.

Winslow, J. T., Noble, P. L., Lyons, C. K. Sterk, S. M., and Insel, T. R. (2003) Rearing effects on cerebrospinal fluid oxytocin concentration and social buffering in rhesus monkeys. Neuropsychopharmacology 28 , 910-918

Young, L. J., Lim, M. M., Gingrich, B., and Insel, T. R. (2001) Cellular mechanisms of social attachment. Horm. Behav. 40, 133-138.

Young, L. J., and Wang, Z. (2004). The neurobiology of pair bonding. Nat. Neurosci. 7, 1048-1054.

Yu, G. Z., Kaba, H., Okutani, F., Takahashi, S., and Higuchi, T. (1996). The olfactory bulb: a critical site of action for oxytocin in the induction of maternal behaviour in the rat. Neuroscience 72, 1083-1088.

Ziegler, T. E., Scheffler, G., and Snowdon, C. T. (1995). The relationship of cortisol levels to social environment and reproductive functioning in female cotton-top tamarins, Saguinus oedipus. Horm. Behav. 29, 407-424.

Conflict of Interest Statement: The authors declare that the research was conducted in the absence of any commercial or financial relationships that could be construed as a potential conflict of interest.

Received: 23 December 2011; paper pending published: 30 January 2012; accepted: 10 February 2012; published online: 28 February 2012.

Citation: Nagasawa M, Okabe S, Mogi $K$ and Kikusui $T$ (2012) Oxytocin and mutual communication in motherinfant bonding. Front. Hum. Neurosci. 6:31. doi: 10.3389/fnhum.2012.00031 Copyright (c) 2012 Nagasawa, Okabe, Mogi and Kikusui. This is an openaccess article distributed under the terms of the Creative Commons Attribution Non Commercial License, which permits non-commercial use, distribution, and reproduction in other forums, provided the original authors and source are credited. 